\title{
LE PIANTE SONO DESTRE O MANCINE?
}

\author{
Nota del s.c. CARLO SOAVE (*)
}

(Adunanza del 25 giugno 2015)

SunTO. - Tutte le piante presentano movimenti oscillatori degli organi in crescita denominati "circumnutazione". Sono movimenti che, associati all'allungamento, descrivono nello spazio spirali circolari. In Arabidopsis thaliana il senso di rotazione della circumnutazione è destrorso ed è geneticamente determinato da un singolo gene con due alleli: l'allele "destrorso" dominante sull'allele "sinistrorso". Lo stesso avviene in Zea mays dove ancora un singolo gene controlla la spiralizzazione destrorsa o sinistrorsa dei semi sulla spiga. Inoltre è stato possibile determinare che la specificazione dell'asimmetria destra/sinistra dipende in Zea mays dalla determinazione dell'asse antero-posteriore della spiga.

$* * *$

ABSTRACT. - Circumnutation is an oscillating movement of a growing plant organ that results from an endogenous rhythmic process intrinsic to growth. Circumnutating organs, as they extend, describe a helical trace. In Arabidopsis thaliana circumnutation is particularly evident in primary roots and occurs, as in most plants, in a right-handed direction. In $A$. thaliana the direction of circumnutation depends on the presence of a single gene with the right-handed allele dominant over the left -handed, as in Zea mays where again a single gene controls the left or right spiralization of the kernels on the ear. Furthermore we demonstrate that the specification of the left/right asymmetry is dependent on the establishement of the antero-posterior axis on the ear.

Le piante, a differenza degli animali, non vanno in giro per il mondo, a meno che qualcuno le porti. Ma, proprio perchè sono abbar-

(") Istituto Lombardo Accademia di Scienze e Lettere; Università degli Studi di Milano, Italy. E-mail: carlo.soave@unimi.it 
bicate al terreno hanno sviluppato la capacità di operare numerosi movimenti che permettono di adattarsi alle varie condizioni ambientali. Sentono la direzione della forza di gravità per cui le radici crescono verso il basso e gli apici vegetativi verso l'alto, seguono il movimento del sole e fuggono dall'ombra. Ma c'è un altro movimento che Charles Darwin, nel volume "The power of movement in plants" scritto insieme al figlio Francis e pubblicato nel 1880 da John Murray (London), chiama circumnutation (circumnutazione) [1]. Ecco come Darwin descrive questo movimento: "Se noi guardiamo un apice vegetativo di una pianta quando emerge dal terreno, vedremo che all'inizio esso piega verso nord, poi gradualmente si volge a est e poi a sud, a ovest e infine ancora a nord. Se il movimento è stato regolare, l'apice avrà descritto un cerchio, ma siccome contemporaneamente cresce verso l'alto, più che un cerchio sarà una spirale circolare. Questo movimento è stato chiamato da Sachs "revolving nutation", ma noi troviamo più conveniente denominarlo "circumnutation".

Tutte le piante (e non solo le piante, ma anche i funghi, le alghe e alcuni batteri coloniali) durante la fase di crescita "circumnutano", sia a livello dell'apice vegetativo che a livello dell'apice radicale e questo movimento serve a esplorare l'ambiente in cui si trovano. Per esempio, nelle piante rampicanti la circumnutazione fà sì che i viticci trovino l'appoggio solido attorno al quale avvolgersi. Perchè ci sia circumnutazione deve esserci obbligatoriamente crescita in quanto il movimento oscillatorio è intrinsecamente dipendente dall'allungamento cellulare. Una cellula vegetale aumenta le sue dimensioni principalmente per distensione secondo l'asse longitudinale della cellula. Questo avviene perchè essa è ricoperta da una parete formata da fibre di cellulosa disposte in spire che avvolgono la cellula trasversalmente all'asse longitudinale. Ne consegue che la pressione idrostatica presente all'interno della cellula è contrastata in senso radiale dai legami covalenti, molto forti, tra i monomeri della cellulosa, mentre in senso longitudinale è contrastata dai legami idrogeno, più deboli, che tengono unite le spire una sopra l'altra: la conseguenza è che fino a che c'è sufficiente pressione idrostatica interna, la cellula si allunga. Se l'allungamento fosse identico su tutti i fianchi dell'organo in crescita, avremmo una crescita diritta, ma se l'allungamento fosse più elevato per esempio sul lato destro, allora l'organo piegherebbe a sinistra; viceversa per un allungamento più elevato sul lato sinistro. Nella circumnutazione la zona di allungamento più attiva si muove di continuo in modo circolare su un piano 
trasversale attorno all'asse di crescita dell'organo e, di conseguenza, la combinazione dei due movimenti genera una spirale circolare. Guardando dal retro nella direzione dell'asse di crescita, la direzione del movimento circolare può essere destrorsa o sinistrorsa, ma nella maggioranza delle piante essa è destrorsa come la direzione di una vite che si avvita [2].

Arabidopsis thaliana è una piccolissima crucifera che da qualche anno è diventata la pianta modello per gli studi di genetica vegetale per il suo breve ciclo vitale (due mesi), l'abbondante produzione di semi, la facilità di coltivazione in laboratorio e il suo genoma, piccolo e completamente sequenziato. Essa però è una pianta completa e, come tutte le altre piante, circumnuta. Il senso di rotazione della radice primaria si può facilmente evidenziare facendo crescere $A$. thaliana in agar supplementato con sali minerali in piastre Petri. Quando la radice, crescendo verso il basso, tocca il fondo della piastra, l'allungamento gravitropico è impedito, il passo della spirale si accorcia e si formano cerchi concentrici. E' facile allora vedere che il senso di rotazione è destrorso. (Fig. 1).

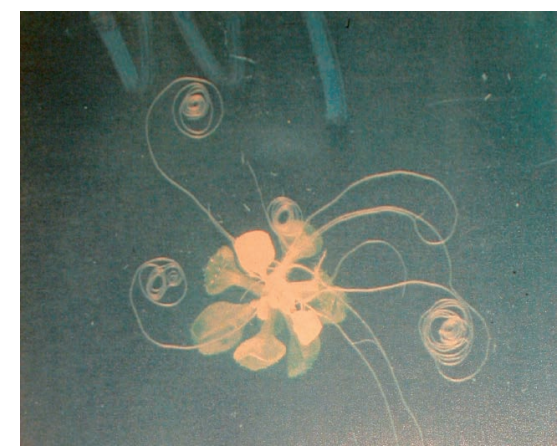

Fig. 1 - Circumnutazione nella radice di A. thaliana.

In natura esistono evidenze che il senso di rotazione destrorso o sinistrorso è geneticamente determinato. Nella chiocciola di acqua dolce Limnea peregra per esempio ci sono esemplari con avvolgimento destrorso o sinistrorso del guscio e il senso di avvolgimento è determinato da un singolo gene con due alleli la cui espressione è controllata per via materna. In $A$. thaliana non esistono evidenze al riguardo. Tuttavia in una popolazione di piantine sottoposte a un trattamento mutageno con etilmetansulfonato è emerso un individuo in cui la circumnutazione radicale appariva sinistrorsa invece che destrorsa (Fig. 2). Nella prima genera- 
zione filiale dell'incrocio tra una pianta con circumnutazione destrorsa e una sinistrorsa, tutta la progenie appariva destrorsa e, nella progenie dopo autofecondazione della prima generazione filiale (seconda generazione) si trovavano piante con circumnutazione destrorsa e sinistrorsa in rapporti numerici significativamente non diversi da 3 a 1 . Ulteriori esperimenti di reincrocio della prima generazione filiale con piante parentali o incroci reciproci (piante destrorse o sinistrorse utilizzate come donatore di polline) dimostravano che il carattere rotazione dell'apice radicale (e anche dell'apice vegetativo) si comportava in $A$. thaliana in modo perfettamente mendeliano, cioè un singolo gene con due stati possibili (alleli) controlla il senso di rotazione: l'allele destrorso, dominante, induce rotazione destrorsa, l'allele sinistrorso rotazione nel senso opposto.

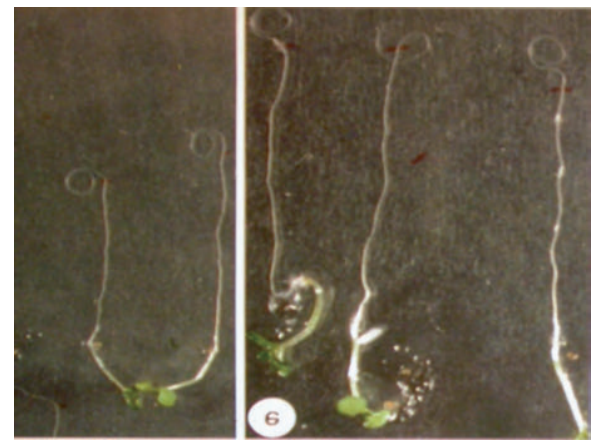

Fig. 2 - Circumnutazione sinistrorsa e destrorsa della radice di piante di A. thaliana allevate sulla superficie di piastre di agar.

Il problema biologico di fondo comunque è da dove la pianta trae l'informazione per individuare il lato destro rispetto al sinistro. Indicazioni al proposito vengono da studi sugli animali dotati di simmetria bilaterale. Questi animali hanno tre assi corporei: un asse anteroposteriore, un asse dorso-ventrale e un asse sinistro-destro. La questione centrale è quando e dove viene specificato e determinato l'asse sinistro-destro e sopratutto se la sua determinazione sia dipendente o indipendente dalla determinazione degli altri due assi. Nel primo caso se l'orientamento di un asse cambia, anche l'orientamento degli altri due assi dovrebbe modificarsi; nel caso di indipendenza invece l'orientamento di uno degli assi dovrebbe essere disaccoppiato dall'orientamento degli altri assi. Evidenze al proposito esistono nel riccio di mare Litechinus variegatus: durante lo sviluppo embrionale della larva di $L$. 
variegatus è possibile infatti revertire sperimentalmente l'orientamento dell'asse dorso-ventrale e in conseguenza di questa reversione si osserva reversione anche dell'asse sinistro-destro [3]. Questo risultato dimostra che l'asimmetria dell'asse sinistro-destro è specificata rispetto all'asse dorso-ventrale.

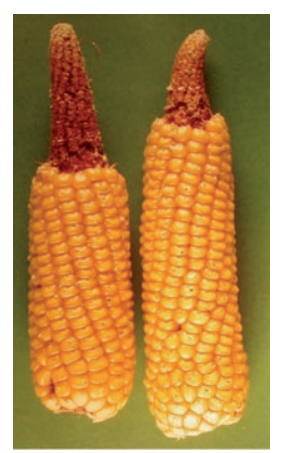

Fig. 3 - Ranghi sinistrorsi e destrorsi in spighe di mais.

Questo esperimento non è replicabile in A. thaliana in quanto non si evidenzia nella radice (e neppure nella pianta intera) un asse dorso-ventrale: l'unico asse riconoscibile è quello apice radicale-apice vegetativo e, al momento, non ci è noto nessun approccio sperimentale che possa cambiarne l'orientamento. Un'alternativa è offerta dalla pianta di mais Zea mays, dove nella spiga esiste un asse antero-posteriore evidenziato dal fatto che l'embrione di tutti i semi posti sulla spiga è rivolto verso l'apice. Esiste inoltre una mutazione, denominata reversed germ orientation ( $r g o$ ), in cui l'orientamento dell'embrione è opposto, è rivolto verso la base della spiga e non verso l'apice. Inoltre nella collezione di linee pure di mais conservate presso la sezione di maiscoltura (Stezzano, Bergamo) dell'Istituto Sperimentale per la Ceralicoltura, sono state trovate due spighe in cui i ranghi dei semi sono disposti in spirale rispettivamente destrorsa e sinistrorsa (Fig. 3). Anche in questo caso l'incrocio tra la linea con ranghi destrorsi e quella con ranghi sinistrorsi ha prodotto spighe della prima generazione tutte con spirale destrorsa e, nella generazione successiva, si è osservata una segregazione di spighe destrorse $v s$ spighe sinistrorse in rapporto $3: 1$. Il risultato è quello atteso per un singolo gene che controlla la spiralizzazione con due stati allelici, destrorso dominante su sinistrorso, come in $A$. thaliana. A questo punto è possibile ripetere in mais l'esperimento di Mc 
Cain e Mc Clay [3] su L. variegatus e cioè verificare se la specificazione dell'asse sinistro-destro nella spiga di mais è dipendente dalla determinazione dell'asse antero-posteriore della spiga. Infatti se, tramite successivi incroci, spighe a spirale destrorsa sono introgredite in un background genetico reversed germ orientation (e quindi ribaltando l'asse antero-posteriore), le nuove spighe rgo presentano spirale sinistrorsa. L'opposto si verifica se spighe sinistrorse sono introgredite in background genetico con normale orientamento dell'asse antero-posteriore.

Si è dimostrato così che anche in una specie vegetale la specificazione di uno degli assi del corpo non è indipendente dalla specificazione di almeno un altro degli assi corporei.

\section{BIBLIOGRAFIA}

1. Darwin CA, Darwin F, The Power of Movement in Plants, John Murray, London, 1880.

2. Baillaud L, Les mouvements d'exploration et d'enroulement des plantes volubiles, in: Encyclopedia of plant physiology, vol XVII; Physiology of movements, part 2, Ruhland H, ed. Springer, pp 637-715 Berlin, 1962.

3. McCain ER, McClay DR, The establishment of bilateral asymmetry in sea urchin embryos, Development 1994:120: 395-404. 